\title{
A review of perioperative anaphylaxis at a Singapore tertiary hospital
}

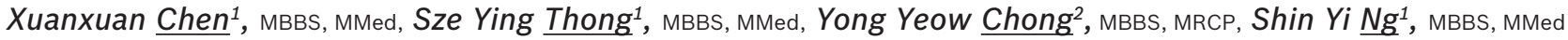

INTRODUCTION Perioperative anaphylaxis is an anaesthetic emergency, but its incidence is not well described in the local literature. This retrospective study aims to look at a group of patients who had perioperative anaphylaxis in our institution. METHODS We conducted a retrospective review of electronic databases and clinical case sheets, and identified 34 patients who had possible perioperative anaphylaxis during anaesthesia in our institution between 1 January 2007 and 30 April 2012. RESULTS After reviewing clinical and biochemical data, we found that 16 out of 151,876 patients who underwent surgery had confirmed perioperative anaphylaxis, an incidence of 1:10,000. Neuromuscular blockers were identified as the most common causative agent for perioperative anaphylaxis. The offending agent could not be identified in seven patients. CONCLUSION To the best of our knowledge, this is the first study in Southeast Asia on the incidence of perioperative anaphylaxis. Having a preoperative history of allergy did not seem to predispose patients to the subsequent development of perioperative anaphylaxis.

Keywords: allergy, anaphylaxis, perioperative

\section{INTRODUCTION}

Perioperative anaphylaxis is a rare event that is associated with morbidity and mortality. Its diagnosis is challenging and requires close collaboration between the anaesthesiologist and the allergist in order to identify causative agents and prevent the recurrence of similar events. The incidence of severe allergic reactions and offending allergens in each institution differs according to demographics, genetics, anaesthetic practices, changes in practice and the prevalence of a drug in a country. While occurrences of perioperative anaphylaxis are well described in many international case series, ${ }^{(1,2,3)}$ its incidence in Singapore is unknown. Hence, we conducted a study to define the incidence and characteristics of the event in our institution.

\section{METHODS}

This was a retrospective study on patients at Singapore General Hospital, Singapore, with possible perioperative anaphylaxis during anaesthesia between 1 January 2007 and 30 April 2012. During this period, surgery was performed on 151,876 patients at our institution, of which 126,915 operations were performed in the major operating theatre and 24,961 in the ambulatory surgery centre. The patients included in our study met one or more of the following conditions: (a) referred to the allergy clinic; (b) discharge summary had the diagnosis 'anaphylaxis', 'anaphylactoid', 'hypersensitivity' and 'allergy'; (c) serum tryptase test was performed; and (d) had anaphylaxis reported in the institution's risk management system, a voluntary incident-reporting database. Following approval by the SingHealth Centralised Institutional Review Board, we reviewed the medical records of all patients who met the inclusion criteria.

Pertinent data was collected, such as patient demographics (including date of birth, gender and race), medical history, details of the perioperative anaphylaxis events and subsequent investigations. Medical history included allergy (type of allergic reaction and specific allergen), as well as medical, surgical and anaesthesia history. Details of the events surrounding the diagnosis of perioperative anaphylaxis included the date, nature and urgency of the surgery; American Society of Anesthesiologists (ASA) class, clinical manifestations and outcome of the patient; phase of anaesthesia during the adverse event; suspected drugs and their time of administration; and management of the adverse event. The reaction was graded from I-IV in terms of increasing severity (Grade I: presence of cutaneous signs; Grade II: presence of measurable but not life-threatening symptoms, including cutaneous effects, arterial hypotension (defined as a fall in measured blood pressure by $\geq 30 \%$ associated with unexplained tachycardia), cough or difficulty in achieving mechanical ventilation; Grade III: presence of life-threatening reactions: cardiovascular collapse, tachycardia or bradycardia, arrhythmias or severe bronchospasm; Grade IV: circulatory inefficacy, cardiac and/or respiratory arrest)..$^{(1)}$

The results of patients who underwent serum tryptase tests were collected. A serum tryptase level of $>11.4 \mu \mathrm{g} / \mathrm{L}$ was considered elevated by our laboratory standards. An allergist analysed the results of patients who underwent skin prick tests and intradermal tests and categorised them into positive and negative results. Patients suspected to have perioperative anaphylaxis were analysed to determine if they met the Brighton Collaboration case definition for anaphylaxis. ${ }^{(4)}$

For all levels of diagnostic certainty, anaphylaxis was defined as a clinical syndrome characterised by sudden onset and rapid progression of signs and symptoms, and involving multiple $(\geq 2)$ organ systems. This was subdivided into three levels: Level 1 consisted of $\geq 1$ major dermatological and $\geq 1$ major

${ }_{1}^{1}$ Department of Anaesthesiology, ${ }^{2}$ Department of Rheumatology and Immunology, Singapore General Hospital, Singapore

Correspondence: Dr Xuanxuan Chen, Associate Consultant, Department of Anaesthesiology, Singapore General Hospital, Outram Road, Singapore 169608.

chen.xuanxuan@singhealth.com.sg 
cardiovascular and/or respiratory criteria; Level 2 consisted of $\geq 1$ major cardiovascular and respiratory criteria, or $\geq 1$ major cardiovascular/respiratory and $\geq 1$ minor criteria involving $\geq 1$ different systems (other than cardiovascular/respiratory systems), or $\geq 1$ major dermatological and $\geq 1$ minor cardiovascular and/ or minor respiratory criteria; and Level 3 consisted of $\geq 1$ minor cardiovascular/respiratory criterion and $\geq 1$ minor criterion from each of $\geq 2$ different systems/categories. Major criteria were subdivided into dermatological/mucosal, cardiovascular and respiratory. Dermatological/mucosal presentations consisted of generalised urticaria (hives) or generalised erythema, angioedema (not hereditary angioedema) that could be localised or generalised, or generalised pruritus with skin rash. Cardiovascular presentations consisted of hypotension, based on measurement, and clinical diagnosis of uncompensated shock, indicated by a combination of at least three of the following: tachycardia, capillary refill time $>3$ seconds, reduced central pulse volume, and decreased level or loss of consciousness. Respiratory presentations consisted of bilateral wheezing (bronchospasms), stridor, upper airway swelling (lip, tongue, throat, uvula or larynx) and respiratory distress consisting of two or more of the following: tachypnoea, increased use of accessory respiratory muscles (sternocleidomastoid, intercostals, etc), recession, grunting and cyanosis. Minor criteria were subdivided into dermatological/mucosal, cardiovascular, respiratory, gastrointestinal and laboratory features. Dermatological/mucosal presentations consisted of generalised pruritus without skin rash, generalised prickling sensation, localised urticaria at injection site, and red and itchy eyes. Cardiovascular presentations consisted of reduced peripheral circulation, as indicated by a combination of at least two of the following: tachycardia, a capillary refill time $>3$ seconds without hypotension and decreased level of consciousness. Respiratory presentations consisted of a persistent dry cough, hoarse voice, difficulty breathing without wheezing/stridor, the sensation of throat closure, sneezing and rhinorrhoea. Gastrointestinal presentations consisted of diarrhoea, abdominal pain, nausea and vomiting. Laboratory presentations consisted of mast cell tryptase levels of more than the normal upper limit.

The data was studied for the presence of major cardiovascular, respiratory and dermatological features as well as minor cardiovascular, respiratory, dermatological, gastrointestinal and laboratory features. Patients were then classified into three groups based on the level of diagnostic certainty. Fig. 1 shows a summary of the study protocol. Statistical analysis was conducted using SPSS version 16.0 for Windows (SPSS Inc, Chicago, IL, USA).

\section{RESULTS}

A total of 34 patients who underwent surgery during the study period were identified to have possible perioperative anaphylaxis. Of these 34 patients, 16 (seven male, nine female) patients had confirmed perioperative anaphylaxis, based on the Brighton Collaboration case definition used in this study. The other 18 patients were excluded due to missing data or inadequate major or minor clinical manifestations. The overall incidence of

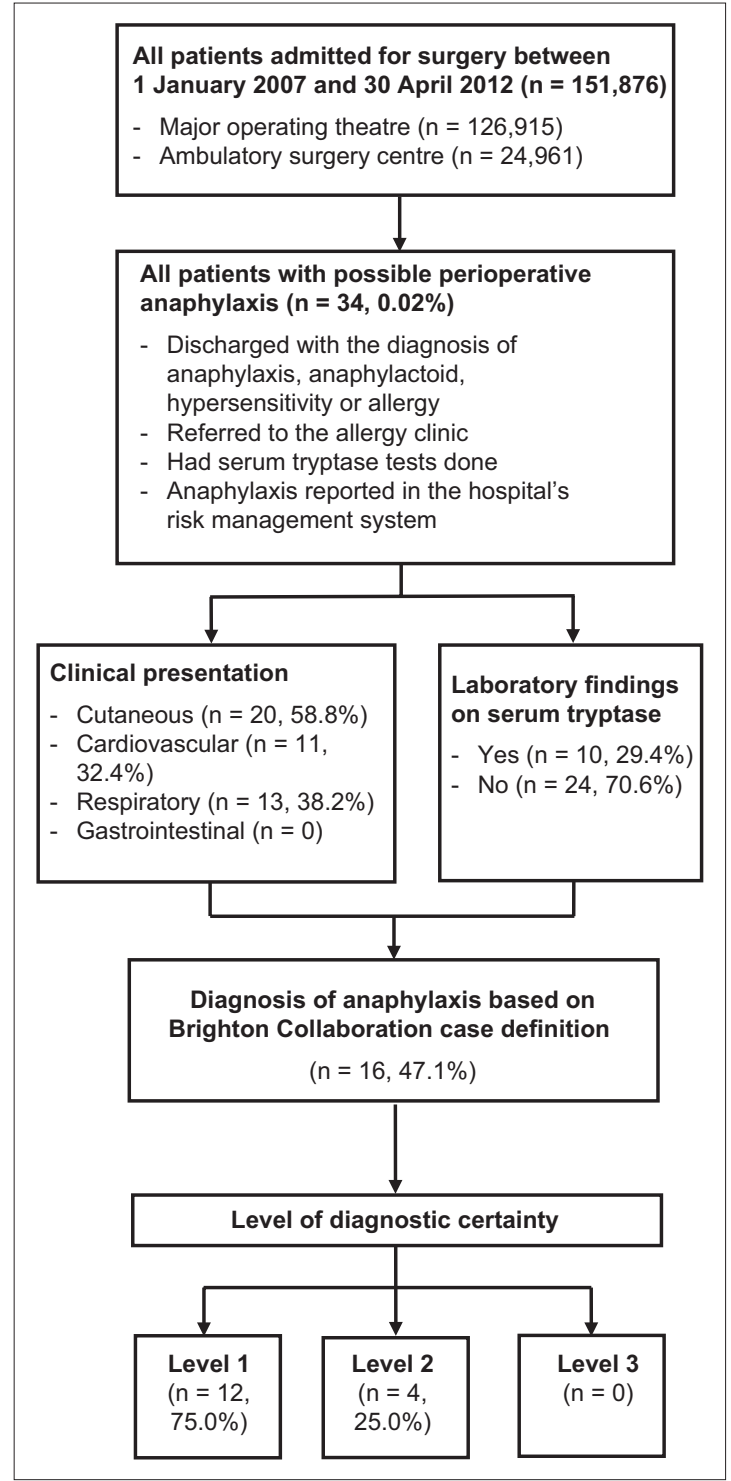

Fig. 1 Flowchart shows the study protocol.

confirmed perioperative anaphylaxis in our study was $0.011 \%$. These patients were predominantly Chinese $(87.5 \%, \mathrm{n}=14)$. Of the 16 patients with confirmed perioperative anaphylaxis, $8(50.0 \%)$ had a history of allergy; $5(31.3 \%)$ patients had only drug allergies, $2(12.5 \%)$ patients had only food allergies, and $1(6.3 \%)$ patient had a combination of drug and food allergies. Patients with a drug allergy were most commonly allergic to antibiotics $(18.8 \%, \mathrm{n}=3)$, followed by nonsteroidal antiinflammatory drugs $(12.5 \%, \mathrm{n}=2)$, opioids $(12.5 \%, \mathrm{n}=2)$, paracetamol $(6.3 \%, \mathrm{n}=1)$ and other classes of drugs $(6.3 \%, \mathrm{n}=1)$.

The medical history of these patients was examined and $6(37.5 \%)$ patients had a history of cardiovascular disease. Respiratory and genitourinary disease, seasonal allergies and previous anaphylaxis were each present in $1(6.3 \%)$ patient. Of these patients, $8(50.0 \%)$ had previously undergone anaesthesia; it was uneventful in all cases. The patient characteristics are summarised in Table I. Most of the patients who had possible perioperative anaphylaxis were ASA II patients $(56.3 \%, \mathrm{n}=9$ ) and were admitted for non-emergency surgery $(93.8 \%, \mathrm{n}=15)$ (Table II). 
Table I. Characteristics of patients with possible and confirmed perioperative anaphylaxis.

\begin{tabular}{|c|c|c|}
\hline \multirow[t]{2}{*}{ Characteristic } & \multicolumn{2}{|c|}{ No. (\%) } \\
\hline & $\begin{array}{c}\text { Possible } \\
\text { cases }(n=34)\end{array}$ & $\begin{array}{c}\text { Confirmed } \\
\text { cases }(n=16)\end{array}$ \\
\hline \multicolumn{3}{|l|}{ Gender } \\
\hline Male & $12(35.3)$ & $7(43.8)$ \\
\hline Female & $22(64.7)$ & $9(56.3)$ \\
\hline \multicolumn{3}{|l|}{ Race } \\
\hline Chinese & $26(76.5)$ & $14(87.5)$ \\
\hline Malay & $4(11.8)$ & 0 \\
\hline Indian & $2(5.9)$ & 0 \\
\hline Caucasian & 0 & 0 \\
\hline Other & $2(5.9)$ & $2(12.5)$ \\
\hline History of allergy* & $18(52.9)$ & $8(50.0)$ \\
\hline \multicolumn{3}{|l|}{ Type of allergen ${ }^{+}$} \\
\hline Drug & $19(55.9)$ & $6(37.5)$ \\
\hline NSAID & $6(17.6)$ & $2(12.5)$ \\
\hline Paracetamol & $4(11.8)$ & $1(6.3)$ \\
\hline Anarex & $1(2.9)$ & 0 \\
\hline Opioid & $2(5.9)$ & $2(12.5)$ \\
\hline Midazolam & $1(2.9)$ & $1(6.3)$ \\
\hline Antibiotics & $13(38.2)$ & $3(18.8)$ \\
\hline Other & $6(17.6)$ & $1(6.3)$ \\
\hline Latex & 0 & 0 \\
\hline Food & $3(8.8)$ & $3(18.8)$ \\
\hline Adhesive & 0 & 0 \\
\hline \multicolumn{3}{|l|}{ Medical history } \\
\hline Respiratory (e.g. asthma) & $5(14.7)$ & $1(6.3)$ \\
\hline Genitourinary & $7(20.6)$ & $1(6.3)$ \\
\hline $\begin{array}{l}\text { Central nervous system } \\
\text { disease }\end{array}$ & $1(2.9)$ & 0 \\
\hline Diabetes mellitus & $1(2.9)$ & 0 \\
\hline $\begin{array}{l}\text { Dermatological } \\
\text { (e.g. eczema, urticaria) }\end{array}$ & 0 & 0 \\
\hline $\begin{array}{l}\text { Seasonal allergies (e.g. hay } \\
\text { fever, allergic rhinitis) }\end{array}$ & $2(5.9)$ & $1(6.3)$ \\
\hline Previous anaphylaxis & $1(2.9)$ & $1(6.3)$ \\
\hline Cardiovascular disease & $13(38.2)$ & $6(37.5)$ \\
\hline \multicolumn{3}{|l|}{ Surgical history } \\
\hline Yes & $23(67.6)$ & $8(50.0)$ \\
\hline General anaesthesia & $21(67.6)$ & $6(37.5)$ \\
\hline Regional anaesthesia & $1(2.9)$ & $1(6.3)$ \\
\hline Monitored anaesthesia care & $1(2.9)$ & $1(6.3)$ \\
\hline No & $11(32.4)$ & $8(50.0)$ \\
\hline \multicolumn{3}{|l|}{ ASA status } \\
\hline 1 & $8(23.5)$ & $4(25.0)$ \\
\hline II & $19(55.9)$ & $9(56.3)$ \\
\hline III & $7(20.6)$ & $3(18.8)$ \\
\hline IV & 0 & 0 \\
\hline
\end{tabular}

* Not all patients had a history of allergy. tPatients could have been allergic to more than one drug. ASA: American Society of Anesthesiologists; NSAID: Nonsteroidal anti-inflammatory drug

The characteristics of the perioperative anaphylaxis cases are shown in Table III. The most common clinical presentation among
Table II. Types of surgery performed on patients with possible and confirmed perioperative anaphylaxis.

\begin{tabular}{lcc}
\hline Characteristic & \multicolumn{2}{c}{ No. (\%) } \\
\cline { 2 - 3 } & $\begin{array}{c}\text { Possible } \\
\text { cases }(\mathbf{n}=\mathbf{3 4})\end{array}$ & $\begin{array}{c}\text { Confirmed } \\
\text { cases }(\mathbf{n}=\mathbf{1 6})\end{array}$ \\
\hline Emergency & $4(11.8)$ & $1(6.3)$ \\
Yes & $30(88.2)$ & $15(93.8)$ \\
No & & \\
Type of surgery* & $13(38.2)$ & $9(56.3)$ \\
General & $5(14.7)$ & $2(12.5)$ \\
Orthopaedic & $4(11.8)$ & 0 \\
Head and neck & $2(5.9)$ & 0 \\
Neurosurgery & $4(11.8)$ & $1(6.3)$ \\
Obstetrics and gynaecology & $2(5.9)$ & $2(12.5)$ \\
Cardiothoracic & & \\
\hline
\end{tabular}

* Not all patients had surgery.

Table III. Characteristics of possible and confirmed perioperative anaphylaxis cases.

\begin{tabular}{|c|c|c|}
\hline \multirow[t]{2}{*}{ Parameter } & \multicolumn{2}{|c|}{ No. (\%) } \\
\hline & $\begin{array}{c}\text { Possible } \\
\text { cases }(n=34)\end{array}$ & $\begin{array}{c}\text { Confirmed } \\
\text { cases }(n=16)\end{array}$ \\
\hline \multicolumn{3}{|l|}{ Presentation } \\
\hline Hypotension & $9(26.5)$ & $10(62.5)$ \\
\hline Cardiac arrest & $2(5.9)$ & 0 \\
\hline Bronchospasm & $12(35.3)$ & $10(62.5)$ \\
\hline Angioedema & $11(32.4)$ & $8(50.0)$ \\
\hline Urticaria & $9(26.5)$ & $3(18.8)$ \\
\hline Other & $8(23.5)$ & $11(68.8)$ \\
\hline \multicolumn{3}{|l|}{ Severity (grade) } \\
\hline I & $11(32.4)$ & 0 \\
\hline II & $12(35.3)$ & $8(50.0)$ \\
\hline III & $8(23.5)$ & $8(50.0)$ \\
\hline IV & $1(2.9)$ & 0 \\
\hline \multicolumn{3}{|c|}{ Phase of anaesthesia } \\
\hline Induction & $17(50.0)$ & $9(56.3)$ \\
\hline Maintenance & $2(5.9)$ & $2(12.5)$ \\
\hline Recovery & $3(8.8)$ & $1(6.3)$ \\
\hline Postoperative & $5(14.7)$ & $1(6.3)$ \\
\hline Unknown & $7(20.6)$ & $3(18.8)$ \\
\hline
\end{tabular}

the confirmed cases was hypotension $(62.5 \%, \mathrm{n}=10)$, defined as a fall in measured blood pressure by $\geq 30 \%$ associated with unexplained tachycardia, and bronchospasm $(62.5 \%, \mathrm{n}=10)$; $8(50.0 \%)$ patients had angioedema and $3(18.8 \%)$ had urticaria (generalised hives). None of the confirmed cases of perioperative anaphylaxis presented with cardiac arrest; 11 (68.8\%) patients had other presentations such as tachycardia, bradycardia, shortness of breath, desaturation, flushing and pruritus. Interestingly, two patients who presented with cardiac arrest were listed as cases of possible perioperative anaphylaxis. One patient presented with pulseless electrical activity (PEA); the initial impression was of an allergy to antibiotics or atracurium. He was sent to the intensive care unit after surgery and his serum tryptase levels were requested. He did not fit the criteria for anaphylaxis, as PEA 
was his only manifestation and his serum tryptase results were unavailable. The other patient, who also presented with PEA, was evaluated by an allergist and tested negative on skin prick and intradermal tests for agents that had been used intraoperatively. Serum tryptase levels were not requested in this case. The patient also did not fulfil the criteria for anaphylaxis due to the absence of other manifestations.

Patients were classified into four grades of severity, with Grade I being the least severe and Grade IV being the most severe. For patients with confirmed perioperative anaphylaxis, 8 (50.0\%) were Grade II and 8 (50.0\%) were Grade III. Among the confirmed cases, the majority of the reactions $(56.3 \%, \mathrm{n}=9)$ occurred during induction; 2 (12.5\%) occurred during maintenance, $1(6.3 \%)$ during recovery and 1 (6.3\%) postoperatively. In most cases, reviews of the patients' anaesthetic charts revealed a temporal sequence between the administration of the suspected offending agents and the development of the clinical picture of anaphylaxis. Most of these patients developed signs and symptoms of anaphylaxis within 30 minutes after administration of the suspected agents.

Of the patients with confirmed perioperative anaphylaxis, most were suspected to have anaphylaxis as a result of receiving opioids $(81.3 \%, \mathrm{n}=13)$, followed by antibiotics $(68.8 \%, \mathrm{n}=11)$, anaesthetic agents $(62.5 \%, \mathrm{n}=10)$ and neuromuscular blockers $(50.0 \%, \mathrm{n}=8)$ (Table IV). Advanced cardiac life support measures were not required in most of the possible cases of perioperative anaphylaxis. Of the patients with confirmed perioperative anaphylaxis requiring these measures, 3 (18.8\%) required intubation and $1(6.3 \%)$ required cardiopulmonary resuscitation. The most commonly used drug in the management of confirmed perioperative anaphylaxis was steroids $(81.3 \%, \mathrm{n}=13)$, followed by adrenaline $(62.5 \%, \mathrm{n}=10)$, phenylephrine $(37.5 \%, \mathrm{n}=6)$, salbutamol $(37.5 \%, \mathrm{n}=6)$, antihistamine $(25.0 \%, \mathrm{n}=4)$ ephedrine $(12.5 \%, \mathrm{n}=2)$ and atropine $(12.5 \%, \mathrm{n}=2)$.

The majority $(56.3 \%, \mathrm{n}=9)$ of the patients with confirmed perioperative anaphylaxis were sent to the intensive care unit after the event (Table V). Of these nine patients, seven cases were of Grade III severity and the other two were of Grade II severity (Table III). Due to the lack of clear criteria for the postoperative disposition of such patients, the decision was left to the discretion of the managing anaesthesiologist based on the clinical situation at that time. The postoperative dispositions of the patients are summarised in Table V. No deaths were reported in our series and all the patients recovered well from the event.

Of the 16 confirmed cases, peri-event serum tryptase levels of only nine patients were checked; 5 (31.3\%) were positive and $4(25.0 \%)$ were negative (Table VI). By our laboratory standards, a positive serum tryptase level was defined as serum tryptase $>11.4 \mu \mathrm{g} / \mathrm{L}$. A skin prick test was performed for $13(81.3 \%)$ of the confirmed cases and an intradermal test was performed for $11(68.8 \%)$ of them. These results were analysed by the allergist following the event. All 13 patients had negative skin prick tests, and 8 (50.0\%) had positive and $3(18.8 \%)$ had negative intradermal tests.

Among the 34 patients with possible perioperative anaphylaxis, $16(47.1 \%)$ patients had confirmed perioperative
Table IV. Drugs suspected to be responsible for possible and confirmed perioperative anaphylaxis cases and the resuscitation measures used.

\begin{tabular}{|c|c|c|}
\hline \multirow[t]{2}{*}{ Parameter } & \multicolumn{2}{|c|}{ No. (\%) } \\
\hline & $\begin{array}{c}\text { Possible } \\
\text { cases }(n=34)\end{array}$ & $\begin{array}{c}\text { Confirmed } \\
\text { cases }(n=16)\end{array}$ \\
\hline \multicolumn{3}{|l|}{ Suspected drug* } \\
\hline Neuromuscular blocker & $14(41.2)$ & $8(50.0)$ \\
\hline Opioid & $23(67.6)$ & $13(81.3)$ \\
\hline Antibiotics & $16(47.1)$ & $11(68.8)$ \\
\hline Anaesthetic agent & $16(47.1)$ & $10(62.5)$ \\
\hline Local anaesthetic & $9(26.5)$ & $5(31.3)$ \\
\hline Latex & $1(2.9)$ & $1(6.3)$ \\
\hline Colloid & $1(2.9)$ & 0 \\
\hline Blood product & $2(5.9)$ & $1(6.3)$ \\
\hline Other & $11(32.4)$ & $5(31.3)$ \\
\hline Missing data & $3(8.8)$ & $1(6.3)$ \\
\hline \multicolumn{3}{|c|}{ Advanced cardiac life support } \\
\hline $\begin{array}{l}\text { Cardiopulmonary } \\
\text { resuscitation }\end{array}$ & $3(8.8)$ & $1(6.3)$ \\
\hline Cardioversion & 0 & 0 \\
\hline Intubation & $3(8.8)$ & $3(18.8)$ \\
\hline None & $28(82.4)$ & $12(75.0)$ \\
\hline \multicolumn{3}{|l|}{ Resuscitation drug* } \\
\hline Adrenaline & $11(32.4)$ & $10(62.5)$ \\
\hline Ephedrine & $3(8.8)$ & $2(12.5)$ \\
\hline Phenylephrine & $9(26.5)$ & $6(37.5)$ \\
\hline Antihistamine & $9(26.5)$ & $4(25.0)$ \\
\hline Steroid & $17(50.0)$ & $13(81.3)$ \\
\hline Salbutamol & $7(20.5)$ & $6(37.5)$ \\
\hline Atropine & $3(8.8)$ & $2(12.5)$ \\
\hline
\end{tabular}

*More than one drug may have been used per person.

Table V. Postoperative dispositions of patients with possible and confirmed perioperative anaphylaxis.

\begin{tabular}{lcc}
\hline Location & \multicolumn{2}{c}{ No. (\%) } \\
\cline { 2 - 3 } & $\begin{array}{c}\text { Possible } \\
\text { cases }(\mathbf{n}=\mathbf{3 4})\end{array}$ & $\begin{array}{c}\text { Confirmed } \\
\text { cases }(\mathbf{n}=\mathbf{1 6})\end{array}$ \\
\hline Intensive care unit & $12(35.3)$ & $9(56.3)$ \\
High dependency unit & $4(11.8)$ & $1(6.3)$ \\
General ward & $10(29.4)$ & $4(25.0)$ \\
Outpatient care & $3(8.8)$ & $1(6.3)$ \\
(appointment, ED) & $5(14.7)$ & $1(6.3)$ \\
Unknown & & \\
\hline
\end{tabular}

ED: emergency department

anaphylaxis based on the Brighton Collaboration case definition. The diagnostic certainty of $12(75.0 \%)$ patients was classified as Level 1, 4 (25.0\%) patients as Level 2 and none as Level 3. 18 patients with possible perioperative anaphylaxis eventually did not meet the case definition for perioperative anaphylaxis. Of the patients with confirmed perioperative anaphylaxis, a causative agent could not be found for 7 (43.8\%) of them (Table VII). For cases in which an agent was identified, neuromuscular blockers were the most common causative agent, with $6(37.5 \%)$ patients identified as being allergic to them. The next most common 
Table VI. Laboratory tests of patients with possible and confirmed perioperative anaphylaxis.

\begin{tabular}{|c|c|c|}
\hline \multirow[t]{2}{*}{ Parameter } & \multicolumn{2}{|c|}{ No. (\%) } \\
\hline & $\begin{array}{c}\text { Possible } \\
\text { cases }(n=34)\end{array}$ & $\begin{array}{c}\text { Confirmed } \\
\text { cases }(n=16)\end{array}$ \\
\hline \multicolumn{3}{|l|}{ Serum tryptase test } \\
\hline Done & $10(29.4)$ & $9(56.3)$ \\
\hline Positive & $5(14.7)$ & 5 (31.3) \\
\hline Negative & $5(14.7)$ & $4(25.0)$ \\
\hline Not done & $24(70.6)$ & $7(43.8)$ \\
\hline \multicolumn{3}{|l|}{ Skin prick test } \\
\hline Done & $27(79.4)$ & $13(81.3)$ \\
\hline Positive & $2(5.9)$ & 0 \\
\hline Negative & $25(73.5)$ & $13(81.3)$ \\
\hline Not done & $6(17.6)$ & $3(18.8)$ \\
\hline Missing data & $1(2.9)$ & 0 \\
\hline \multicolumn{3}{|l|}{ Intradermal test } \\
\hline Done & $21(61.8)$ & $11(68.8)$ \\
\hline Positive & $14(41.2)$ & $8(50.0)$ \\
\hline Negative & $7(20.6)$ & $3(18.8)$ \\
\hline Not done & $11(32.4)$ & $4(25.0)$ \\
\hline Missing data & $2(5.9)$ & $1(6.3)$ \\
\hline \multicolumn{3}{|l|}{$\begin{array}{l}\text { Meets case definition of } \\
\text { anaphylaxis }\end{array}$} \\
\hline Yes (level of evidence) & $16(47.1)$ & $16(100.0)$ \\
\hline 1 & $12(35.3)$ & $12(75.0)$ \\
\hline 2 & $4(11.8)$ & $4(25.0)$ \\
\hline 3 & 0 & 0 \\
\hline No & $18(52.9)$ & 0 \\
\hline
\end{tabular}

Table VII. Causative agents responsible for confirmed perioperative anaphylaxis $(n=16)$.

\begin{tabular}{ll}
\hline Causative agent $^{*}$ & No. (\%) \\
\hline Antibiotics & $3(18.8)$ \\
Cephalosporin & $1(6.3)$ \\
Penicillin & \\
Neuromuscular blocker & $2(12.5)$ \\
Atracurium & $2(12.5)$ \\
Pancuronium & $1(6.3)$ \\
Rocuronium & $1(6.3)$ \\
Succinylcholine & \\
Opioid & $2(12.5)$ \\
Morphine & $1(6.3)$ \\
Pethidine & $1(6.3)$ \\
Chlorhexidine & $7(43.8)$ \\
None found & \\
\hline
\end{tabular}

* More than one causative agent was responsible in some cases.

causative agent was antibiotics, with $4(25.0 \%)$ patients. $3(18.8 \%)$ patients were allergic to opioids, $1(6.3 \%)$ to chlorhexidine and $1(6.3 \%)$ to both morphine and cephalosporin.

\section{DISCUSSION}

This study found that 16 out of 151,876 patients $(0.011 \%$ ) at our institution had perioperative anaphylaxis over a six-year period. There was a greater proportion of women (56.3\%) with perioperative anaphylaxis compared to men (43.8\%) (Table I). This female predominance was also seen in previous studies. A French study showed a female-to-male ratio of 2.7:1 for anaphylactic reactions. ${ }^{(1)}$ In a Norwegian study, the female-to-male ratio for anaphylaxis during anaesthesia was 3:1.(2) The higher incidence of females with anaphylaxis could be due to cross-reactivity to cosmetics, hair care products and household cleaning agents, which women are more commonly exposed to compared with men. These products contain the quaternary ammonium group, also found in neuromuscular blocking agents. ${ }^{(5)}$

Of the patients with confirmed perioperative anaphylaxis, $50.0 \%$ had a history of allergy, the same incidence as those who did not. Therefore, the presence of a preoperative allergy did not seem to result in an increased risk of perioperative anaphylaxis. The rate was higher than that reported in a French study, in which $25.4 \%$ of patients who had perioperative anaphylaxis had a history of atopy. ${ }^{(1)}$ In the present study, $37.5 \%$ of the patients with confirmed perioperative anaphylaxis had a history of drug allergy. This rate was again higher than that reported in the French study (15.9\%). ${ }^{(1)}$ Among the confirmed cases, 3 (18.8\%) patients had a history of food allergies and $2(11.1 \%)$ of them had allergies to both drugs and food. In the group falsely suspected to have perioperative anaphylaxis, 9 (50.0\%) patients had a history of drug allergies; none of them had a history of food allergies or a history of allergies to both drugs and food. The data seems to suggest that having a history of drug allergies did not predispose the patients to perioperative anaphylaxis, but that a history of food allergies did.

Other atopic conditions such as asthma and hay fever were only present in two of the patients with confirmed perioperative anaphylaxis. It was found that four other asthmatic patients who were suspected cases and one patient who had hay fever did not have confirmed perioperative anaphylaxis. This data seems to suggest that the presence of a previous allergy or atopic conditions did not predispose our patients to perioperative anaphylaxis. Most of the cases with perioperative anaphylaxis were identified during the induction phase of anaesthesia. This was also the case in the Norwegian study by Harboe et al. ${ }^{(2)}$ It may be because many drugs are usually given in quick succession during induction. Hence, anaphylaxis is most commonly identified in the induction phase and commonly used agents are often implicated in the peri-induction stage.

Presentations of anaphylaxis are sudden and rapid, involving multiple organ systems. ${ }^{(4)}$ Diagnosis is based on clinical manifestations. The use of laboratory investigations such as the serum tryptase test helps to support the diagnosis of anaphylaxis; a rise in serum tryptase levels following an anaphylactic reaction is indicative, as mast cells release tryptase during an anaphylactic reaction. However, it should be noted that a negative serum tryptase result does not exclude anaphylaxis. Serum tryptase levels peak 1-2 hours after the onset of symptoms of anaphylaxis. Therefore, the timing of the sample collection is important for accurate results. Serum tryptase levels were not routinely measured in patients who were suspected to have perioperative 
anaphylaxis, with only $10(29.4 \%)$ patients being tested. Of these, a positive result was seen in $5(31.3 \%)$ patients with confirmed perioperative anaphylaxis, but a negative result did not exclude the diagnosis of perioperative anaphylaxis, as 4 (25.0\%) patients had negative results but were diagnosed with confirmed perioperative anaphylaxis. The diagnosis of anaphylaxis was not based on serologic findings alone. According to the case definition of anaphylaxis, 16 patients eventually had confirmed perioperative anaphylaxis. Based on the results of this study, it is difficult to conclude if serum tryptase levels correlate with the severity of the clinical presentation. Serum tryptase levels did not seem to correlate with skin test results: two patients had positive tryptase levels but negative skin prick or intradermal tests, and two patients had negative tryptase levels but positive skin tests.

In patients with confirmed perioperative anaphylaxis, despite further investigations, no identifiable causative agent was found in $7(43.8 \%)$ cases (Table VII), as they did not have positive skin prick or intradermal tests. Neuromuscular blockers were the most common causative agents (37.5\%), followed by antibiotics (25.0\%). The finding that neuromuscular blockers were the most commonly implicated agents in perioperative anaphylaxis was similar to that of previous studies. ${ }^{(1-3)}$ Although the majority of patients with possible perioperative anaphylaxis $(n=12)$ were admitted to the intensive care unit (Table $\mathrm{V}$ ), their outcomes were good. This may be due to early recognition and prompt treatment.

A few cases were of particular interest. In the group of patients with confirmed perioperative anaphylaxis, one female patient with a history of crab allergy presented with hypotension, bronchospasm and flushing. She was managed with adrenaline and steroids, then sent postoperatively to the intensive care unit. The patient's serum tryptase levels were positive, but subsequent skin prick and intradermal tests were negative and no causative agent was identified. Another female patient with no history of allergy also presented with hypotension and bronchospasm. She was managed with adrenaline, steroids and salbutamol. She was sent to the intensive care unit postoperatively. Her serum tryptase result was negative and subsequent skin prick and intradermal tests were also negative. No causative agent was identified.

As seen in these patients, anaphylaxis involves multiple systems and a diagnosis can be established regardless of serum tryptase results. Despite best efforts to identify the causative agent, it may not be found. In the group of patients in whom perioperative anaphylaxis was suspected but not found, cutaneous manifestations of angioedema and urticaria were the most common presentations. One patient presented with only angioedema post-induction and was managed with steroids. The patient was sent to the general ward after surgery. A serum tryptase test was not performed and skin prick tests were negative, but intradermal tests were positive for morphine. Hence, even though the patient did not meet the case definition of anaphylaxis, the patient was found to be allergic to morphine. However, the reaction did not amount to anaphylaxis. Two other patients presented solely with PEA requiring cardiopulmonary resuscitation and the use of adrenaline. The serum tryptase test was not performed for one patient, and subsequent skin prick tests and intradermal tests were negative. The other patient had serum tryptase tested, but no results were available. Similarly, no results were available for the skin prick and intradermal tests. Even though the presentations of these two patients were drastic, they only involved the cardiovascular system and the patients did not meet the criteria for anaphylaxis. Due to the myriad of manifestations of anaphylaxis, the differential diagnosis that accompanies these manifestations and the varied test results that accompany the workup, diagnosis and management of anaphylaxis remain a challenge. Due to a high index of suspicion and prompt administration of supportive measures, none of the patients in the study died.

Despite the shortcomings inherent to retrospective studies, this is the first study in Southeast Asia on the incidence of perioperative anaphylaxis and can provide valuable information on perioperative anaphylaxis in this region. It looked at a large patient pool over six years and eventually included 34 patients who had possible perioperative anaphylaxis. The study found that the incidence of perioperative anaphylaxis was 1:10,000. Neuromuscular blockers were the most common causative agent, and a preoperative history of allergy did not seem to predispose patients to develop perioperative anaphylaxis.

\section{REFERENCES}

1. Laxenaire MC, Mertes PM; Groupe d'Etudes des Réactions Anaphylactoïdes Peranesthésiques. Anaphylaxis during anaesthesia. Results of a two-year survey in France. Br J Anaesth 2001; 87:549-58.

2. Harboe T, Guttormsen AB, Irgens A, Dybendal T, Florvaag E. Anaphylaxis during anesthesia in Norway: a 6-year single-center follow-up study. Anesthesiology 2005; 102:897-903.

3. Chong YY, Caballero MR, Lukawska J, Dugué P. Anaphylaxis during general anaesthesia: one-year survey from a British allergy clinic. Singapore Med J 2008; 49:483-7.

4. Rüggeberg JU, Gold MS, Bayas JM, et al; Brighton Collaboration Anaphylaxis Working Group. Anaphylaxis: case definition and guidelines for data collection, analysis, and presentation of immunization safety data. Vaccine 2007; 25:5675-84.

5. Mertes PM, Demoly P, Stenger R. Diseases Summaries. Allergy to Anesthetic Agents. In: World Allergy Organization [online]. Available at: http://www.worldallergy.org/professional/allergic_diseases_center/ anaesthetic_agents/. Accessed February 25, 2016. 